\title{
Erratum to: The effects of spiritual intervention and changes in dopamine receptor gene expression in breast cancer patients
}

\author{
Mohammad Esmael Akbari ${ }^{1}$ - Farah Lotfi Kashani ${ }^{1}$ - Ghasem Ahangari ${ }^{2}$. \\ Majid Pornour $^{2,3} \cdot$ Hessam Hejazi $^{2,4} \cdot$ Elah Nooshinfar $^{1} \cdot$ Mohsen Kabiri $^{5}$. \\ Leili Hosseini ${ }^{1}$
}

Published online: 30 December 2015

(C) The Japanese Breast Cancer Society 2015

\section{Erratum to: Breast Cancer \\ DOI 10.1007/s12282-015-0658-z}

Unfortunately, the author's affiliations have been incorrectly published in the original publication of the article.

The correct affiliations are provided in this erratum.

The online version of the original article can be found under doi:10.1007/s12282-015-0658-z.

Leili Hosseini

leili.hosseini@gmail.com

1 Cancer Research Center, Shahid Beheshti University of Medical Sciences, Tehran, Iran

2 Department of Medical Genetics, National Institute of Genetic Engineering and Biotechnology, Tehran, Iran

3 Medical Laser Research Center, ACECR, Tehran, Iran

4 Department of Biology, Faculty of Science, Lorestan University of Medical Sciences, Khoramabad, Iran

5 Department of Language, Aryanpour Institute, Tehran, Iran 